\title{
CARACTERIZAÇÃO DE UM NOVO CAROTENÓIDE MINORITÁRIO DE URUCUM ${ }^{1}$
}

\author{
Adriana Zerlotti MERCADANTE ${ }^{2, *}$, Hanspeter PFANDER ${ }^{3}$
}

\begin{abstract}
RESUMO
Entre os corantes naturais, o urucum é o mais utilizado no Brasil. Entretanto, o emprego de seus extratos tem sofrido restrições no que se refere à quantidade permitida bem como à gama de produtos a que pode ser adicionado. Uma das alegações é que pouco se conhece sobre a composição do urucum e de suas preparações. No presente estudo um novo carotenóide foi isolado de sementes de urucum em quantidades traços e purificado por cromatografia semi-preparativa em coluna aberta, camada delgada e líquida de alta eficiência. A sua estrutura foi parcialmente elucidada através das informações combinadas provenientes dos espectros no UV/visível, de massas e de ressonância magnética nuclear de próton, incluindo técnicas bidimensionais. Este é o primeiro relato da ocorrência de um álcool de alto peso molecular ligado ao carotenóide (9'Z)-apo-6'-licopenóico.
\end{abstract}

Palavras-chave: carotenóide; urucum; estrutura química.

\section{SUMMARY}

CHARACTERIZATION OF A NEW MINOR CAROTENOID FROM ANNATTO. Among the natural colorants, annatto is the most used in Brazil. However, the use of annatto extracts has been under control in relation to the amount allowed and the varieties of products in which the extracts can be added. One of the reasons is the insufficient knowledge about the composition of annatto and its preparations. In the present study, a new carotenoid was isolated in trace amount and purified through semi-preparative column, thin layer and high performance liquid chromatography. Its structure was partially elucidated by the combined information from spectra of UV/visible, mass and proton nuclear magnetic resonance, including two dimensional technique). This is the first report on the occurrence of a high molecular weight alcohol linked to the carotenoid (9'Z)-apo-6'-lycopenoic.

Keywords: carotenoid; annatto; chemical structure.

\section{1 - INTRODUÇÃO}

O urucum é um dos corantes naturais mais utilizados, e o Brasil um dos maiores produtores e exportadores de sementes de urucum. Mudança na ingestão diária aceitável (IDA) para os extratos de urucum ocorreu na década de 80 , pois nos anos 70 [7, 8], a IDA era de $1,25 \mathrm{mg} / \mathrm{kg}$ de peso corpóreo diminuindo para $0,065 \mathrm{mg} / \mathrm{kg}$ de peso corpóreo em 1982 [9]. Além disso, a aplicação de extratos de urucum em alimentos tem sofrido severas restrições no que se refere à gama de produtos a que pode ser adicionado bem como quanto à quantida-

\footnotetext{
${ }_{1}^{1}$ Recebido para publicação em 17/11/00. Aceito para publicação em 28/06/01.

${ }^{2}$ Departamento de Ciência de Alimentos, Faculdade de Engenharia de Alimentos, UNICAMP, Cx. Postal 6121, CEP: 13083970, Campinas, SP. E-mail: mercadan@obelix.unicamp.br.

${ }^{3}$ Department of Chemistry and Biochemistry, University of Berne, Freiestrasse 3, 3012, Berna, Suiça.

${ }^{*}$ A quem a correspondência deve ser enviada.
}

de permitida. Uma das alegações é que pouco se conhece sobre a composição desses extratos.

Bixina é o carotenóide majoritário das sementes de urucum, perfazendo um mínimo de $80 \%$ dos carotenóides totais [17], e é também o carotenóide majoritário de colorífico [18] e das preparações lipossolúveis [19]. Foi o primeiro carotenóide natural identificado a possuir configuração cis (ou Z) [1, 11, 12]. Esta estrutura, Figura 1, com 25 carbonos e grupos carboxílico e éster metílico é muito diferente dos carotenóides usualmente presentes em alimentos (cadeia com 40 carbonos) e foi até o presente momento encontrada somente no urucum.<smiles>CC(=O)OC=CC(C)=CC=CC(C)=CC=CC=C(C)C=CC=C(C)C=CC(=O)O</smiles>

FIGURA 1. Estrutura da bixina.

Outros carotenóides, em quantidades muito pequenas, foram isolados de sementes de urucum e suas estruturas foram elucidadas através de técnicas espectroscópicas como espectrometria de massas e ressonância magnética nuclear (RMN). Estes novos carotenóides puderam ser divididos em três grupos: ésteres metílicos de apocarotenóides $\left(C_{30}\right.$ e $\left.C_{32}\right)$ [13, 14], diapocarotenóides com grupos terminais éster metílico, cetona e aldeído $\left(C_{19}, C_{22}, C_{24}\right.$ e $\left.C_{25}\right)[10,15]$ e ácidos de diapocarotenóides $\left(\mathrm{C}_{22}\right.$ e $\left.\mathrm{C}_{24}\right)$ esterificados com grupos metil e geranilgeraniol [16]. Além desses carotenóides, um carotenóide-derivativo com 14 carbonos foi isolado [15] e a presença de fitoeno, fitoflueno, $\zeta$-caroteno e neurosporeno, todos com 40 carbonos, foi confirmada [13].

Assim, o presente estudo teve como objetivo isolar e caracterizar um novo carotenóide minoritário presente nas sementes de urucum.

\section{2 - MATERIAL E MÉTODOS}

\section{1 - Material}

Sementes de urucum foram adquiridas em Campinas, Brasil.

\section{2 - Extração e isolamento}

O carotenóide minoritário foi isolado a partir de $1 \mathrm{~kg}$ de sementes segundo metodologia descrita por MERCADANTE et al [14]. Após extração com acetato de etila e éter t-metil butílico em ausência de luz por cerca de 24 horas, as fases orgânicas foram combina- 
das e levadas até secura em evaporador rotatório $\left(\mathrm{T}<30^{\circ} \mathrm{C}\right)$, resultando em $94 \mathrm{~g}$ de uma resina oleosa de cor vermelha escura.

O extrato foi aplicado em coluna de alumina neutra entre grau III e IV $(10 \mathrm{~cm}$ de diâmetro e $12 \mathrm{~cm}$ de altura) de onde a primeira fração foi eluída com hexano/éter t-metil butílico (1:1) e os carotenóides com grupo ácido, como bixina, permaneceram no topo da coluna. Após concentração, a primeira fração $(21 \mathrm{~g})$ foi submetida à cromatografia em coluna de sílica-gel $(6 \mathrm{~cm}$ de diâmetro e $37 \mathrm{~cm}$ de comprimento) [14]. A fração eluída da coluna de sílica com $100 \%$ tolueno $(0,7 \mathrm{~g}$, com aspecto oleoso) foi a escolhida para prosseguir o isolamento. Esta fração foi a seguir purificada por cromatografia em camada delgada (CCD) deMgO/kieselguhr (1:1) $(0,5 \mathrm{~mm})$ com acetona/hexano (2:3) como fase móvel. Seis bandas foram separadas e a principal, com coloração rosa e $R_{f} 0,1$, foi raspada e o carotenóide eluído com acetato de etila.

Para a purificação final utilizou-se um cromatógrafo líquido de alta eficiência (CLAE) com sistema ternário de bombeamento de solvente Kontron (modelo T-414), detetor de arranjo de diodos (Waters, modelo 990) e válvula injetora tipo "Rheodyne" com alça de amostra de $100 \mu \mathrm{L}$ de capacidade. A separação foi realizada em coluna semi-preparativa $\mathrm{C}_{18}$ Nucleosil (Macherey-Nagel), com $3 \mu \mathrm{m}$ de diâmetro de partícula, $250 \mathrm{~mm}$ de comprimento e $10 \mathrm{~mm}$ de diâmetro interno; e como fase móvel metanol/acetato de etila/éter t-metil butílico (8:1:1) com fluxo de $3 \mathrm{~mL} / \mathrm{min}$. Nestas condições, o carotenóide eluiu com $t_{R}$ de $15 \mathrm{~min}$, resultando em $0,6 \mathrm{mg}$ de pigmento purificado.

\section{3 - Caracterização}

Com o carotenóide purificado foram medidos os espectros de UV/visível, de massas e de RMN.

\subsection{1 - Equipamentos}

O espectro no UV-visível foi obtido em espectrofotômetro Perkin Elmer, modelo lambda 6, em éter t-metil butílico. O grau de estrutura fina do espectro foi expresso como \%III/II [2].

Os espectros de massas foram obtidos com um equipamento VG (VG Biotech., UK), modelo Quattro Triple Quadrupole. Os carotenóides foram diretamente introduzidos por sonda na câmara de ionização a $240^{\circ} \mathrm{C}$, utilizando ionização por impacto de elétrons, com energia de $70 \mathrm{eV}$. A interpretação dos espectros de massas foi baseada nas fragmentações características, comparadas com as fornecidas na literatura [5].

Para os experimentos de RNM utilizou-se um equipamento Bruker DRX-500. As determinações foram feitas sob argônio, a $23^{\circ} \mathrm{C}$, em clorofórmio deuterado (99,95\%) eluído previamente por uma mini-coluna de alumina. Antes da análise por RNM, traços de solventes das amostras foram removidos sob alto vácuo. Os deslocamentos químicos $(\delta)$ foram relacionados com o sinal residual do solvente e as designações referentes aos prótons foram alcançadas através dos experimentos ${ }^{1} \mathrm{H}$ e H,H-COSY ("correlated spectroscopy"). Como a quantidade de amostra disponível não era suficiente para realizar experimentos que detectam carbono 13 , os valores de $\delta$ dos carbonos ligados aos prótons foram extraídos do experimento que detecta prótons, HMQC ("heteronuclear multiquantum coherence"), otimizado para constante de acoplamento ${ }^{1} \mathrm{~J}_{\mathrm{CH}}$, com precisão de $0,3 \mathrm{ppm}$. Os valores de $\delta$ de ${ }^{1} \mathrm{H} \mathrm{e}{ }^{13} \mathrm{C}$ obtidos foram comparados com os fornecidos na literatura [4].

\subsection{2 - Derivatizações químicas}

O carotenóide isolado foi submetido à saponificação com $10 \% \mathrm{KOH}$ em metanol e à redução com $\mathrm{LiAlH}_{4}$ $[3,6]$. Após purificação por CCD de sílica-gel com diclorometano/éter de petróleo, nas proporções 3:2 e $1: 1$ respectivamente, foram medidos os espectros no UV/visível e de massas dos compostos formados.

\section{3 - RESULTADOS E DISCUSSÃO}

A estrutura parcial do carotenóide isolado no presente estudo, éster do (9'Z)-apo-6'-licopenoato (1), bem como as estruturas dos produtos (2 e 3 ) resultantes das derivatizações químicas estão apresentadas na Figura 2.

O carotenóide 1 com 10 duplas ligações conjugadas, uma na forma cis, e uma carbonila conjugada apresentou $\lambda_{\max }$ em $460 \mathrm{~nm}$, espectro com baixa estrutura fina $\mathrm{e}$ um pico cis de baixa intensidade, como mostram os dados da Tabela 1. Como esperado, o espectro no UVI visível do ácido (9'Z)-apo-6'-licopenóico (2), formado após saponificação do carotenóide 1 , apresentou deslocamento hipsocrômico de $3 \mathrm{~nm}$ e um ligeiro aumento na estrutura fina do espectro (Tabela 1). O álcool primário correspondente (carotenóide 3 ) apresentou espectro com deslocamento hipsocrômico de $16 \mathrm{~nm}$ e significativo aumento na estrutura fina do espectro devido à redução da carbonila conjugada à cadeia poliênica no presente carotenóide 1 (Tabela 1). Estes efeitos já haviam sido apresentados para os derivados éster metílico, ácido e álcool de torularrodina [2]. O éster do (9'Z)-apo-6'-licopenoato (1) apresentou comprimentos de onda máximo de absorção no UV/visível e forma do espectro idênticos aos do metil (9'Z)-apo-6'-licopenoato, reportado anteriormente por MERCADANTE et al [13], indicando que ambos possuem o mesmo cromóforo.

O íon molecular do éster do (9'Z)-apo-6'-licopenoato (1) apareceu em $\mathrm{m} / \mathrm{z} 624$, que é compatível com $\mathrm{C}_{44} \mathrm{H}_{64} \mathrm{O}_{2}$. O fragmento a $\mathrm{m} / \mathrm{z} 457[\mathrm{M}-167]^{+}$, de maior intensidade que o íon molecular, indicou a perda do grupo R. Eliminação de xileno da cadeia poliênica combinada com a

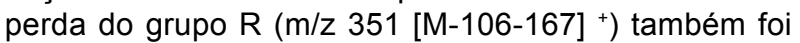
observada (Tabela 1). O ácido 2 resultante da saponificação do carotenóide 1 apresentou íon molecular em $\mathrm{m} / \mathrm{z} 458$, e eliminação de xileno da cadeia poliênica $(\mathrm{m} / \mathrm{z} 352)$. Devido à redução do éster, o álcool 3 formado apresentou íon molecular a $\mathrm{m} / \mathrm{z} 444$, e fragmentos a $\mathrm{m} / \mathrm{z} 426$ e a m/z 338 correspondentes à eliminação de água e xileno, respectivamente (Tabela 1). Com as 
derivatizações empregadas (Figura 2), a presença de uma cadeia de hidrocarboneto $(R)$ com peso molecular de 168 daltons no éster (9'Z)-apo-6'-licopenoato (1) foi confirmada. Além disso, os álcoois obtidos através da redução do metil (9'Z)-apo-6'-licopenoato [13] e do carotenóide 1 apresentaram as mesmas características de $\lambda_{\max }$ e íon molecular, indicando serem o mesmo composto.

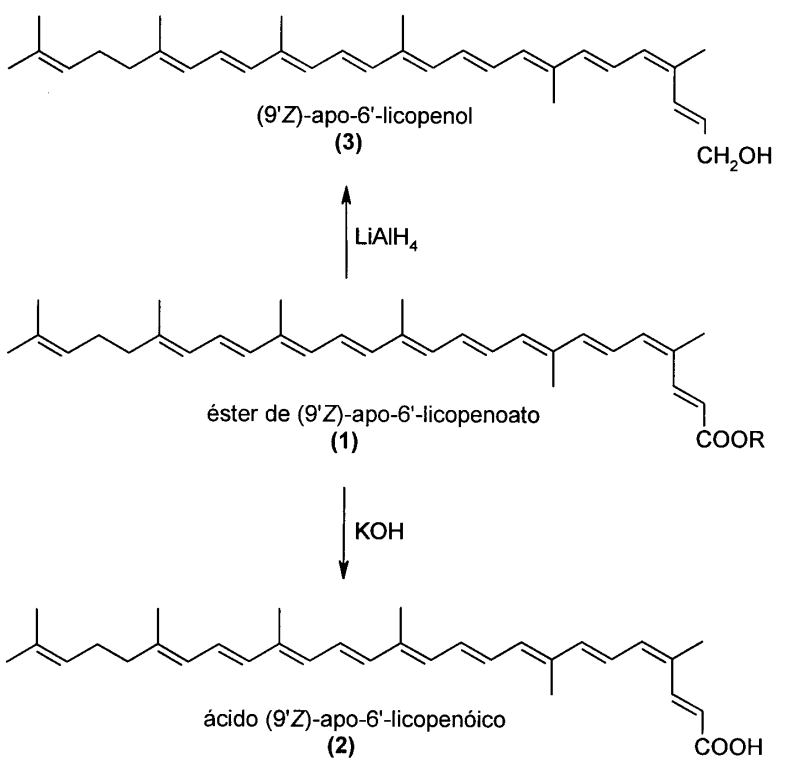

FIGURA 2. Estrutura do novo carotenóide e dos produtos formados após derivatizações.

TABELA 1. Características espectroscópicas do novo carotenóide e dos produtos após derivatizações.

\begin{tabular}{|c|c|c|c|}
\hline Carotenóide & $\lambda_{\max }$ & $\%$ III/III & $m / z(\%)$ \\
\hline éster do (9'Z)-apo-6'- & $340,355,(436), 460,488^{a}$ & 0 & $624\left(\mathrm{M}^{+}, 9\right), 457(\mathrm{M}-167$ \\
\hline licopenoato (1) & $362,(440), 464,488^{\mathrm{b}}$ & 0 & $\begin{array}{l}25), 351 \text { (M-167-106, 63), } \\
69(100)\end{array}$ \\
\hline $\begin{array}{l}\text { éster do }\left(9^{\prime} Z+\text { all-E)-apo-6'- }\right. \\
\text { licopenoato }\end{array}$ & $360,(439), 464,491^{a}$ & 10 & $\begin{array}{l}624\left(M^{+}, 1\right), 457(M-167, \\
3), 351(M-167-106,10), \\
69(100)\end{array}$ \\
\hline ácido apo-6'-licopenóico (2) & $(436), 461,490^{\mathrm{a}}$ & 23 & $\begin{array}{l}458\left(\mathrm{M}^{+}, 13\right), 352(\mathrm{M}-106, \\
18), 91(100)\end{array}$ \\
\hline apo-6'-licopenol (3) & $424,448,478^{\mathrm{a}}$ & 51 & $\begin{array}{l}444\left(\mathrm{M}^{+},<1\right), 338(\mathrm{M}-106, \\
\text { 2), } 69(100)\end{array}$ \\
\hline
\end{tabular}

aSolvente: éter t-metil butílico.

'Solvente: metanol/éter t-metil butílico/acetato de etila (8:1:1).

Através dos espectros de RMN, a parte referente ao carotenóide da estrutura do éster (9'Z)-apo-6'licopenoato (1) foi completamente elucidada (Tabela 2). Os valores de $\delta$ para carbonos quaternários não puderam ser obtidos uma vez que os valores para ${ }^{13} \mathrm{C}$ foram extraídos do experimento bidimensional HMQC que irradia prótons.

A configuração da dupla ligação na forma (9'Z) foi confirmada através dos valores característicos dos deslocamentos de isomerização de ${ }^{1} \mathrm{H} \Delta \delta=\delta_{9^{\prime} \mathrm{z}}-\delta_{\text {all-E }}$ obtidos previamente $[13,14,15,16]$ e que são correspondentes às médias reportadas por ENGLERT [4]. Entretan- to, devido a sinais sobrepostos, até mesmo no espectro bidimensional COSY, a parte referente ao álcool ligado ao ácido de carotenóide não pode ser elucidada.

As formas isoméricas all-E-, (9'Z)- e (7Z,9Z,9'Z)do éster metílico deste mesmo carotenóide foram encontradas em quantidades traços em sementes de urucum por MERCADANTE et al [13, 14]. A presença de um álcool de alto peso molecular, geranilgeranil, ligado a um carotenóide foi relatada pela primeira em 1999 [16].

\section{4 - CONCLUSÕES}

Este é o primeiro relato da ocorrência de um álcool de alto peso molecular ligado ao radical ácido (9'Z)apo-6'-licopenóico.

O novo carotenóide isolado possui, assim como a bixina, configuração cis no carbono 9' e clivagem da dupla ligação entre os carbonos 5' e 6'. Aparentemente os carotenóides de cadeia curta, apo- ou diapocarotenóides, são formados por degradação oxidativa dos carotenóides com quarenta carbonos, embora nenhuma evidência detalhada tenha sido relatada em relação à biossíntese de bixina.

TABELA 2. Deslocamentos químicos e constantes de acoplamento, em $\mathrm{CDCl}_{3}$, do carotenóide (1).

\begin{tabular}{|c|c|c|c|}
\hline $\mathrm{H}-\mathrm{n}^{\circ}$ & ${ }^{1} \mathrm{H}$ & $C-n^{\circ}$ & ${ }^{13} \mathrm{C}$ \\
\hline & $\begin{array}{l}\text { deslocamento químico (ppm), } \\
\text { multiplicidade, constante de } \\
\text { acoplamento }(\mathrm{Hz})\end{array}$ & & $\begin{array}{l}\text { deslocamento } \\
\text { químico (ppm) }\end{array}$ \\
\hline $\mathrm{H}-2$ & $5,15, \mathrm{~m}$ & $\mathrm{C}-2$ & 123,7 \\
\hline $\mathrm{H}-3$ & $2,06, m$ & $\mathrm{C}-3$ & 26,6 \\
\hline $\mathrm{H}-4$ & $2,12, \mathrm{~m}$ & C-4 & 39,6 \\
\hline $\mathrm{H}-6$ & $5,96, d(J=10,7)$ & C-6 & 124,5 \\
\hline $\mathrm{H}-7$ & $6,50, \mathrm{dd}(\mathrm{J}=10,7 ; 15,0)$ & $\mathrm{C}-7$ & $\underline{L}^{\mathrm{a}}$ \\
\hline $\mathrm{H}-8$ & $6,25, d(J=15,0)$ & $\mathrm{C}-8$ & 129,9 \\
\hline $\mathrm{H}-10$ & $6,18, d(J=10,8)$ & $\mathrm{C}-10$ & 131,3 \\
\hline $\mathrm{H}-11$ & $6,65, \mathrm{dd}(\mathrm{J}=10,8 ; 13,9)$ & $\mathrm{C}-11$ & 129,4 \\
\hline $\mathrm{H}-12$ & $6,36, d(J=13,9)$ & $\mathrm{C}-12$ & 134,6 \\
\hline $\mathrm{H}-14$ & 6,27 , parte $X$ do sistema $A B M X$ & $\mathrm{C}-14$ & 132,0 \\
\hline $\mathrm{H}-15$ & 6,69 , parte $A$ do sistema $A B M X$ & C-15 & 131,4 \\
\hline $\mathrm{H}-16$ & $1,68, s$ & $\mathrm{C}-16$ & 25,6 \\
\hline $\mathrm{H}-17$ & $1,60, s$ & $\mathrm{C}-17$ & 17,9 \\
\hline $\mathrm{H}-18$ & $1,82, s$ & $\mathrm{C}-18$ & 16,8 \\
\hline $\mathrm{H}-19$ & $1,94, s$ & C-19 & 12,8 \\
\hline $\mathrm{H}-20$ & $1,98, s$ & $\mathrm{C}-20$ & 12,8 \\
\hline $\mathrm{H}-\mathrm{7}^{\prime}$ & $5,91, d(J=15,5)$ & C-7' & 117,6 \\
\hline $\mathrm{H}-8$ & $7,97, d(J=15,5)$ & C-8' & 140,3 \\
\hline H-10' & $6,36, d(J=12,1)$ & C-10' & 137,8 \\
\hline $\mathrm{H}-11$ & $6,84, \mathrm{dd}(\mathrm{J}=12,1 ; 14,7)$ & C-11' & 125,4 \\
\hline H-12' & $6,40, d(J=14,7)$ & C-12' & 140,5 \\
\hline H-14' & 6,31 , parte $M$ do sistema $A B M X$ & C-14' & 134,6 \\
\hline$H-15^{\prime}$ & 6,62 , parte $B$ do sistema ABMX & C-15' & 129,4 \\
\hline H-19' & $1,96, s$ & C-19 & 20,2 \\
\hline $\mathrm{H}-20^{\prime}$ & $1,98, s$ & C-20' & 12,8 \\
\hline
\end{tabular}

aSinal não foi detectado.

\section{5 - REFERÊNCIAS BIBLIOGRÁFICAS}

[1] BARBER, M.S.; HARDISSON, A.; JACKMAN, L.M.; WEEDON, B.C.L. Studies in nuclear magnetic resonance. Part IV: Stereochemistry of the bixin. J. Chem Soc., p. 1625-1630, 1961. 
[2] BRITTON, G. UV/Visible Spectroscopy. In: BRITTON, G.; LIAAEN-JENSEN, S.; PFANDER, H. (Eds.) Carotenoids: Spectroscopy. Basel: Birkhäuser, 1995. vol 1B, p. 13-62.

[3] DAVIES, B.H. Carotenoids. In: GOODWIN, T.W. (Ed.) Chemistry and Biochemistry of Plant Pigments. London: Academic Press, 1976. Vol 2, p. 38-165.

[4] ENGLERT, G. NMR Spectroscopy. In: BRITTON, G.; LIAAENJENSEN, S.; PFANDER, H. (Eds.) Carotenoids: Spectroscopy. Basel: Birkhäuser, 1995. vol 1B, p. 147-260.

[5] ENZELL, C.R.; BACK, S. Mass Spectrometry. In: BRITTON, G.; LIAAEN-JENSEN, S.; PFANDER, H. (Eds.) Carotenoids: Spectroscopy. Basel: Birkhäuser, 1995. vol 1B, p. 261-320.

[6] EUGSTER, C.H. Chemical Derivatization: Microscale Tests for the Presence of Common Functional Groups in Carotenoids. In: BRITTON, G.; LIAAEN-JENSEN, S.; PFANDER, H. (Eds.) Carotenoids: Isolation and Analysis. Basel: Birkhäuser, 1995. vol 1A, p. 71-80.

[7] FAO/WHO. 1970. Food Additive Series 70.36. Geneva.

[8] FAO/WHO. 1976. Food Additives Serie 7. Geneva, 111 p.

[9] FAO/WHO. 1982. Food and Nutrition Paper 25. Rome. p. 22.

[10] JONDIKO, I.J.O.; PATTENDEN, G. Terpenoids and an apocarotenoid from seeds of Bixa orellana. Phytochem., v. 28, p. 3159-3162, 1989.

[11] KARRER, P.; HELFENSTEIN, A.; WIDMER, R.; VAN ITALLIE, T.B. Uber bixin (XIII Mitteillung uber Pflanzenfarbstoffe). Helv. Chim. Acta, v. 12, p. 741-755, 1929.

[12] KUHN, P.; WINTERSTEIN, A. Uber konjugiente doppelbindugen. XXIII. Die dihydrover-bindung des isomeren bixine und die elektronenkonfiguration der polyene. Ber. Dtsch. Chem. Ges., v. 65, p. 646-651, 1932.

[13] MERCADANTE, A.Z.; STECK, A.; RODRIGUEZ-AMAYA, D.B; PFANDER, H.; BRITTON, G. Isolation of methyl 9'Z-apo6'-lycopenoate from Bixa orellana. Phytochem., v. 41, p. 1201-1203, 1996.

[14] MERCADANTE, A.Z.; STECK, A.; PFANDER, H. Isolation and identification of new apocarotenoids from annatto (Bixa orellana L.) seeds. J. Agric. Food Chem., v. 45, p. 10501054, 1997.

[15] MERCADANTE, A.Z.; STECK, A.; PFANDER, H. Isolation and structure elucidation of minor carotenoids from annatto (Bixa orellana L.) seeds. Phytochem., v. 46, p. 1379-1383, 1997.

[16] MERCADANTE, A.Z.; STECK, A.; PFANDER, H. Three minor carotenoids from annatto (Bixa orellana L.) seeds. Phytochem., v. 52, p. 135-139, 1999.

[17] PRESTON, H.D.; RICKARD, M.D. Extraction and chemistry of annatto. Food Chem., v. 5, p. 47-56, 1980.

[18] TOCCHINI, L.; MERCADANTE, A. Z. Extração e determinação, por CLAE, de bixina e norbixina em colorífico. Ciênc. Tecnol. Aliment., 2001 (aceito).

[19] TOCCHINI, L.; MERCADANTE, A. Z. Teores de bixina e norbixina em preparações lipo-e aquossolúveis de urucum. III Simpósio Latino Americano de Ciência de Alimentos. Campinas, SP, 16-19 de novembro de 1999.

\section{6 - AGRADECIMENTOS}

Os autores agradecem o auxílio financeiro do MCT/ FINEP/PRONEX. 